\title{
Memory for lateral orientation of slides in picture stories
}

\author{
ROBERT N. KRAFT and JAMES J. JENKINS \\ University of Minnesota, Minneapolis, Minnesota 55455
}

\begin{abstract}
Three picture stories were constructed by taking slides of people involved in three different activities. Two sequences of these slides were presented. Subjects were asked whether or not each slide in the second sequence was in the same lateral orientation as in the first sequence. Experiment 1 presented the slides in correct or random sequence and in correct or random lateral orientation ( 2 by 2 design). The two groups who received correct orientation excelled in recognition of orientation in the test series. Experiment 2 replicated the random orientation condition, found that pre-warning of the recognition task had no effect on performance, and suggested that these subjects did not make use of the events in determining laterality. Experiment 3 replicated the correct order and orientation condition and further revealed that new but appropriate slides in the test series were accurately judged as to lateral orientation which fit the context of the original stories.
\end{abstract}

During the past several years the investigation of picture memory has become a lively area of research. Shepard (1967) established that subjects retain enough information from single brief exposures of hundreds of pictures to be able to recognize nearly all of them. Nickerson (1968) determined that there is substantial retention of briefly presented pictorial material up to a year after the original presentation of the material. Standing, Conezio, and Haber (1970) found that after presentation of 2,560 photographic stimuli, recognition performance exceeded $90 \%$ accuracy. However, it has been established more recently that memory for specific types of information contained in the pictures seems to depend upon the nature of the material, the method of presentation and testing, and the type of task the subject performs with the material (Bower \& Karlin, 1974; Madigan \& Rouse, 1974; McCabe \& Jenkins, Note 1). Of particular interest here, some studies have reported that the lateral orientation of a set of pictures was accurately remembered (Standing, et al., 1970; McCabe \& Jenkins, Note 1), whereas other studies have reported that performance on the orientation recognition task was significantly poorer than it would have been on a simple recognition task (Bartlett, 1932;

Preparation of this paper was supported in part by grants to the University of Minnesota, Center for Research in Human Learning, from the National Science Foundation (BNS-75-03016), the National Institute of Child Health and Human Development (HD-01136), the Training Grant (HD-00098), and the Graduate School of the University of Minnesota. Special thanks are due to William $O$. Shaffer and Linda McCabe for their critical suggestions, and Virginia J. Petersen for her necessary encouragement. Requests for reprints should be sent to: Robert Kraft, Center for Research in Human Learning, 205 Elliott Hall, 75 East River Road, University of Minnesota, Minneapolis, Minnesota 55455.
Madigan \& Rouse, 1974; Kraft, Note 2). However, in past studies, memory for the lateral orientation of pictures was usually investigated as a by-product of some other recognition or recall task. The purpose of this study was to investigate directly some important variables which affect memory for lateral orientation of pictures in order to explain some of the apparent discrepancies in the literature.

Bartlett (1932) first reported that subjects could not remember the lateral orientation of faces immediately after each picture was presented. On the orientation test, subjects chose between five possible orientations and were correct only $40 \%$ of the time. It should be noted that the majority of errors were made by choosing the mirror image of the correct orientation. In another study, Standing et al. (1970) found that, although half of the old slides were laterally reversed, recognition performance was as accurate as it was with correctly oriented slides. Thus, it appears that laterally reversing a slide does not adversely affect recognition memory for the slides. However, the same study was repeated with the additional requirement that the subjects report the original orientation of the old slides. The results showed that $30 \mathrm{~min}$ after the presentation, performance was somewhat poorer on the orientation recognition task than on the simple recognition task; however, performance on the orientation task was significantly better than chance. After $24 \mathrm{~h}$, performance on the orientation task dropped significantly and approached chance level, although the subjects could still identify old slides with a high degree of accuracy.

Kraft (Note 2) presented a short acquisition series of 24 easily distinguishable profiles and subsequently presented a test series of 24 old and 24 new profiles. The task was to recognize each slide as familiar or 
unfamiliar and to report the initial orientation of each familiar slide. Those subjects who were informed about the orientation recognition task and who responded "left" or "right" aloud to indicate the direction of each profile in the initial series reported original orientation with only $69 \%$ accuracy. Under all other conditions in the study, subjects performed with less accuracy. Performance on the orientation task was significantly poorer than performance on the simple recognition task, which exceeded $85 \%$ accuracy in all groups. Similarly, Madigan and Rouse (1974) reported that the probability of correct orientation recognition for pictures of common objects was between .69 and .73 , depending on exposure duration, which is significantly lower than performance on the simple recognition task of all the studies mentioned above.

A different line of evidence indicating the difficulty of remembering lateral orientation comes from Klatzky and Stoy (1974), who measured the reaction times of comparisons between identical pictures, mirror image pictures, and pictures of common objects that were physically' different but had the same category name. They found that reaction times for mirror image matches were the same as reaction times for identity matches, whereas reaction times for name matches were significantly longer. Perhaps there was no difference between mirror and identity match reaction times because the mental representations for both types of match were identical. Lateral orientation might not be specified in the mental representation because it is not important in the construction of the events with this particular task and this set of stimuli.

The results above are consistent with the finding of Standing et al. (1970) that laterally reversed slides were recognized as being familiar as easily as identical slides. However, the results above appear to be inconsistent with the finding that the accuracy of orientation memory for laterally reversed slides is quite high.

There are two major methodological differences between those studies that report accurate memory for lateral orientation and those that do not. Bartlett (1932) and Kraft (Note 2) presented only one class of pictorial stimuli: Pictures of faces were used in both studies. Standing and others presented a heterogeneous sample of pictorial stimuli (e.g., city scenes, vegetation, animals, and common objects) in addition to pictures of human beings. Both series of pictures of faces were homogeneous with respect to subject matter, camera distance, type of pose, and perspective, whereas the heterogeneous series of pictures varied with respect to all of these factors. Some of the discrepancy in the results can be explained by a similarity argument which states that orientation memory is poor when the pictorial stimuli are homogeneous with respect to the factors mentioned above, as they were in the studies of Bartlett (1932) and Kraft (Note 2). A parallel example of poorer recognition performance with homogeneous sets of pictures was found by Goldstein and Chance (1971), who demonstrated that even simple recognition of pictures was much poorer when all the stimuli were homogeneous with respect to subject matter.

Second, the kinds of stimuli used in these studies differed with respect to the meaningfulness of the orientation reversal. Laterally reversing slides of people's profiles (Bartlett, 1932; Kraft, Note 2) does not meaningfully alter the stimuli as compared to laterally reversing scenes of a city (Standing et al., 1970). In a real life situation, it matters little to an observer whether a person has his left side toward him or his right side toward him. However, laterally reversing a slide of a city scene would alter the real life consequences of the scene, especially if the observers were instructed to imagine themselves in the situations depicted by the slides. The same reasoning would hold for a slide of a golf club or a baseball glove; in the correct orientation they would be quite functional, but in the laterally reversed orientation they could not be used properly by the observer. A simple functionalist hypothesis might be: If pictorial material consists of stimuli whose function is appreciably altered when laterally reversed, then the orientation of that material will tend to be remembered. If pictorial material consists of stimuli whose function is not appreciably altered when laterally reversed, then the orientation of that material will not tend to be remembered.

The presentation of slides in the form of coherent picture stories should provide the meaningfulness component necessary for lateral orientation to be remembered if lateral orientation is an important aspect of the stories. Coherent picture stories can be constructed by presenting a sequence of slides in the same fashion as a comic strip or photo essay (Baggett, 1975; Jenkins, Wald, \& Pittenger, in press). It is then possible to manipulate the meaningfulness of an orientation reversal, while using the same set of slides in all conditions, by varying the order and orientation of the slides during the initial presentation of these stories. The purpose of Experiment 1 was to manipulate the meaningfulness of the slide presentation in this fashion, in order to determine the extent to which the meaningfulness of the orientation reversals contributes to accuracy of recognition of lateral orientation.

\section{EXPERIMENT 1}

\section{Method}

Subjects. Sixty students enrolled in the introductory psychology course at the University of Minnesota served as subjects in order to receive optional points for the course. There were approximately equal numbers of males and females.

Stimuli and apparatus. Three 10-slide stories were constructed from slides taken of three real life events. The events consisted of one or two people performing a simple activity, with the action moving both to the left and to the right for each story. The three events were as follows: (1) a person learning how to skate, (2) a person taking a box from a shed 
and loading it in a car, and (3) a boy and a girl having a snowball fight. Twenty slides were taken of each real life event and 10 were selected to construct each slide story. No slides were symmetrical about the vertical axis. A Kodak Carousel slide projector was used to project the slides on a white wall.

Procedure and design. Each subject viewed an acquisition series of 30 slides and a test series of the same 30 slides. During the acquisition series, the subjects were instructed to examine each slide carefully but they were not informed about the subsequent recognition test. During the test sequence, subjects were required to report whether each slide was in the same orientation or whether it was laterally reversed with respect to its orientation in the acquisition series. Each subject served in one of four conditions formed by presenting the acquisition series in correct or random order and also correct or random orientation. The design was a 2 (random vs. correct order) by 2 (random vs. correct orientation) between-subjects factorial. The four conditions were as follows: correct context, in which the slides were arranged in the correct orientation and correct order, that is, the slides were arranged as three 10-slide stories; random order context, in which the slides were shown in the same orientation as in the three 10-slide stories, but were arranged in random order; random orientation, in which half the slides were laterally reversed with respect to their orientation in the three 10-slide stories, but the slides were arranged in the correct order; and both random, in which half the slides were laterally reversed with respect to their orientation in the three 10 -slide stories, and the slides were arranged in random order. Each slide in the acquisition series was shown for $5 \mathrm{sec}$, with approximately $1 \mathrm{sec}$ blank time between slides.

The test series was constructed by arranging the 30 slides in a different random order and by laterally reversing half the slides with respect to their correct orientation in the three 10-slide stories and with respect to their orientation in the two random orientation conditions. The slides in the test series were shown for $15 \mathrm{sec}$ each, during which time the subjects made left-right orientation judgments. All subjects received the same test series. As each slide was shown in the test series, the subjects reported whether or not the slide was in the same orientation as it was in the acquisition series.

After the orientation recognition test, the subjects were asked two additional questions. First, they were asked to describe what the people in the slides were doing. Second, they were asked to think of strategies that they had used to remember the orientation of the slides; that is, they were requested to give any intuitions they had about how they remembered orientation.

\section{Results and Discussion}

A summary of the principal results is given in Table 1. The major dependent variable was the percent of correct responses on the orientation recognition task. The 2 by 2 between-subjects design showed one significant main effect, correct vs. random orientation $[F(1,56)=163.18, p<.001]$. For correct vs. random order $[F(1,56)=2.34, p>.10]$, no significant difference was seen in the number of slides correctly recognized. The interaction was not significant $[F(1,56)=.51, p>.25] .($ For all effects, $\mathrm{MSe}=69.47$.)

These results indicate that when the slides in the picture stories were presented in the correct orientation, subjects performed significantly better on the orientation recognition task than when the slides were presented in random orientation. The results also indicate that, with these simple events, the order of the stimuli does not affect the subjects' ability to remember
Table 1

Percent of Correct Responses on the Orientation Recognition Task

\begin{tabular}{cccc}
\hline $\begin{array}{c}\text { Orientation } \\
\text { Of Original } \\
\text { Presentation }\end{array}$ & \multicolumn{2}{c}{ Order of Original Presentation } \\
\cline { 2 - 4 } & Correct & Random & Mean \\
\hline Correct & 95.33 & 93.33 & 94.33 \\
Random & 69.20 & 62.67 & 65.93 \\
Mean & 82.26 & 78.10 & \\
\hline
\end{tabular}

lateral orientation. This finding also has general support in the literature. Bransford and Franks (1971) have shown that subjects are spontaneously able to integrate a long series of nonconsecutive phrases into several sentences which contain more information than any individual phrase. Jenkins and his associates (Jenkins et al., in press) have shown that, even when picture stories are presented out of order, they can specify the nature of the event if the event is intrinsically strongly ordered. Apparently, in this experiment, the subjects in the random order context condition were able to construct coherent events even though the slides were in random order. And, by the same reasoning, subjects in the random orientation condition were not aided by the fact that the slides were in the correct order; the randomly oriented slides gave no coherent flow of movement and thus no basis to form three coherent stories.

In addition to the orientation judgments, the subjects' answers to two posttest questions were analyzed. When asked to describe the activities of the people in the slides, most subjects in all conditions were able to indicate that there were three separate events. However, the detail and accuracy of these descriptions varied between the conditions. Six judges were asked to rate the accuracy and quality of the subjects' descriptions on a scale of 1 to 5 , with a rating of 1 being the lowest and 5 the highest. A summary of the results is given in Table 2. The mean rating for the subjects' descriptions in the two correct orientation conditions was significantly higher than the mean rating for the two random orientation conditions $[F(1,10)=64.02, p<.001]$. Correct vs. random order also showed a significant effect $[F(1,10)=10.17$, $p<.011$, although smaller than the orientation effect (for all effects, $\mathrm{MSe}=.14$ ). The interaction was not significant $[F(1,10)=3.43, p>.05]$. These results suggest that subjects in the two correct orientation conditions were able to describe the underlying stories better than the subjects in the two random orientation conditions. The fact that order also affected the subjects' descriptions may suggest that the correct order subjects described the underlying stories better than the random order subjects, or it may suggest that the correct order subjects were simply better able to distinguish the stories because the slides were grouped together as three separate stories.

When the subjects were asked to list the memory 
Table 2

Mean Judges' Ratings for Accuracy and Quality of Subjects' Descriptions of the Picture Stories: Five-Point Scale

\begin{tabular}{cccc}
\hline $\begin{array}{c}\text { Orientation } \\
\text { Of Original } \\
\text { Presentation }\end{array}$ & \multicolumn{3}{c}{ Order of Original Presentation } \\
\cline { 2 - 4 } & Correct & Random & Mean \\
\hline Correct & 3.79 & 3.08 & 3.44 \\
Random & 2.23 & 2.02 & 2.13 \\
Mean & 3.01 & 2.55 & \\
\hline
\end{tabular}

strategies they used in the orientation recognition task, the strategies fell into three broad categories: (1) Subjects remembered the relation of the principal figure to the background, (2) subjects remembered the orientation of some large salient object, such as a car, and (3) subjects used the direction of movement in the original stories as a cue. The first two strategies involved knowledge of specific features of each particular slide, whereas the third strategy involved knowledge of the underlying events. Once again, six judges were selected to score the difference in strategies between conditions. The judges were told to separate the strategies into two categories: (1) strategies that used underlying movement as a cue, and (2) strategies that used only features of each particular slide as a cue. A summary of the results is given in Table 3. Both main effects were significant [correct vs. random orientation, $F(1,10)=39.89, p<.001$; for correct vs. random order, $F(1,10)=13.11, p<.01]$. The interaction was not significant $[\mathrm{F}(1,10)=.36, \mathrm{p}>.50]$. (For all effects, MSe $=261.12$.) These results indicate that subjects in the two conditions that performed with over $90 \%$ accuracy on the orientation recognition task may have used the underlying movement of the people as a cue, whereas subjects in the two conditions that performed with below $70 \%$ accuracy made little use of and perhaps had little knowledge of the underlying movement. The fact that order had a small effect on the subjects' use of movement cues may simply suggest that the reconstruction of the stories, for the purpose of describing them after the recognition task, may have been enhanced when the slides were presented as three separate stories.

We hypothesize that lateral orientation was remembered accurately in the two correct orientation conditions because the laterally reversed slides in the test series clashed with the meaningful pattern of the original events; that is, for these subjects it made a difference in the total event whether a person was carrying a box to the left or to the right. We suspect that lateral orientation was not remembered in the other two conditions because those subjects could not construct laterally coherent events. Therefore, a slide reversed in the test series could not signal a meaningful alteration. In these last two conditions, we believe that such events as the subjects could construct mainly involved features of each particular slide which remain unchanged after orientation reversal; the events did not involve the direction of movement in the underlying stories, which is appreciably changed after orientation reversal.

An alternative to the meaningful reversal hypothesis would be a simple chunking explanation. This would state that when slides are presented correctly as three separate stories, there are fewer chunks to store and thus the lateral orientation of the slides is remembered better. This hypothesis asserts that the stories are simply mnemonic devices used to chunk together the separate slides with details of each slide remaining separate in memory. However, it is difficult to use this reasoning to explain the superior performance of the random order context group and the poor performance of the random orientation group. The random orientation group received the slides correctly chunked as three 10-slide stories, yet they did not remember lateral orientation with much accuracy. More importantly, the chunking hypothesis is complicated by the fact that the action flows in both directions in each story; therefore, simply grouping slides together according to story would not unambiguously determine the original orientation of the slide.

The results of Experiment 1 suggest that subjects in the correct orientation conditions acquired knowledge of the underlying events and used this knowledge to remember the lateral orientation of the slides. These results also indicate that although subjects in the random orientation conditions performed significantly more poorly than those in the correct orientation conditions, they performed significantly better than chance and, therefore, must have used some cues successfully. The results from Experiment 1 suggest that the random orientation subjects were less able to construct three coherent stories and thus might have had to rely primarily on features of the individual slides in order to remember lateral orientation.

Experiment 2 was designed to determine more directly the extent to which subjects in the random orientation conditions used knowledge of the individual slides to remember lateral orientation. One way to accomplish this was to replicate exactly the both random condition and to compare this group's performance to the performance of a group who received superficially similar slides which had no story connecting them. The results from the second group would indicate the extent to which above chance performance is possible when subjects have only single-

Table 3

Percent of Subjects in Each Condition Judged to Be Using Underlying Movement as a Cue to Orientation

\begin{tabular}{lccc}
\hline $\begin{array}{l}\text { Orientation } \\
\text { Of Original } \\
\text { Presentation }\end{array}$ & \multicolumn{3}{c}{ Order of Original Presentation } \\
\cline { 2 - 4 } & Correct & Random & Mean \\
\hline Correct & 74.45 & 51.12 & 62.78 \\
Random & 33.33 & 8.89 & 21.11 \\
Mean & 53.89 & 30.01 & \\
\hline
\end{tabular}


slide information to draw on. Experiment 2 also employed a control group which was informed concerning the subsequent recognition test. This group, which was otherwise treated identically to the both random group, was included in order to determine whether these subjects could draw on any more effective memory strategies than the incidental both random group.

\section{EXPERIMENT 2}

\section{Method}

Subjects. Forty-five students enrolled in the introductory psychology course at the University of Minnesota served as subjects in order to receive optional points for the course. There were approximately equal numbers of males and females.

Stimuli and apparatus. The intentional both random condition and the incidental both random condition used the same material as the both random condition in Experiment 1. The remaining condition made use of 30 slides taken of people walking across a bridge. Each slide consisted of the side view of one person who was off-center on the slide. There were equal numbers of right and left profiles. In the test series for these three conditions, half the slides were laterally reversed with respect to their orientations in the acquisition series.

Procedure and design. Each subject viewed an acquisition sequence of 30 slides. The incidental both random condition was a replication of the both random condition in Experiment 1. The intentional both random condition was identical to the incidental condition, but the subjects were informed about the subsequent recognition task. The third condition which made use of the 30 unrelated slides was termed the no context condition. It should be noted that since the no context condition made use of a completely different set of slides than were used in the other two conditions, it would be difficult to draw any strong conclusions from the results if the no context group performed differently than the other two conditions. However, if there were no significant difference in the performance of the three groups, then a stronger statement could be made from the results.

\section{Results and Discussion}

A summary of the principal results is given in Table 4 . The major dependent variable was the percent correct responses on the orientation recognition task. As expected, there were no significant differences among the three conditions $[\mathrm{F}(2,42)=.46, \mathrm{p}>.50$, $\mathrm{MSe}=53.36]$. The subjects' performance in the no context condition was the same as the performance in the incidental both random condition, which suggests that the subjects in the both random conditions made no effective use of knowledge of the underlying events as a cue for remembering lateral orientation. Further, informing the subjects did not improve their performance, suggesting that they could not improve

Table 4

Percent of Correct Responses on the Orientation Recognition Task: Experiment 2

\begin{tabular}{ccc}
$\begin{array}{c}\text { Incidental Both } \\
\text { Random }\end{array}$ & $\begin{array}{c}\text { Intentional Both } \\
\text { Random }\end{array}$ & No Context \\
\hline 63.76 & 67.22 & 65.77 \\
\hline
\end{tabular}

their basic strategies, using the information presented to them. In addition, it should be noted that the replicated both random condition yielded results very close to the original both random condition.

Results from the second experiment thus added further support to the suggestion made in Experiment 1 that the story line did not contribute to the performance of the both random subjects in remembering lateral orientation. The other suggestion made earlier that the two correct orientation groups did make use of knowledge of the underlying story line to remember lateral orientation can also be subjected to a more detailed analysis. A third experiment was designed to test more directly whether the correct context subjects in Experiment 1 extracted coherent stories and whether they used knowledge of these stories as a means to remember lateral orientation.

Experiment 3 consisted of two conditions. The first condition was a replication of the correct context condition in Experiment 1. The second condition more directly tested for knowledge of the underlying events by removing some slides from the original test sequence and replacing them with new slides that fit the context of the stories. The subjects in this condition were tested with these new, but appropriate slides. It was hypothesized that the subjects in this condition would infer the correct orientation of the slides from knowledge of the underlying flow of motion in the stories. These new, but appropriate slides were termed "belonging" slides. More specifically, the belonging slides fit in between two temporally contiguous slides in the original stories. It is important to point out that the belonging slides were easily distinguishable from the slides they replaced when viewed simultaneously. The subjects that received the belonging slides were given the same instructions as the second correct context group. They were instructed to report the original orientation; they were not required to distinguish between old and new slides. Thus, correct responses were scored when the old slides were reported in the correct orientation and when belonging slides were reported in the orientation that fit the flow of motion in the original stories. If those subjects that were tested with belonging slides performed as well as subjects who were tested with old slides, then further support would be added to the notion that the subjects in correct orientation conditions were extracting knowledge of the underlying events and were using this knowledge to remember lateral orientation. Both conditions were expected to perform with above $90 \%$ accuracy on orientation judgments.

\section{EXPERIMENT 3}

\section{Method}

Subjects. Thirty students enrolled in the introductory psychology course at the University of Minnesota served as subjects in order to receive optional points for the course. There were approximately equal numbers of males and females. 
Stimuli and apparatus. The slides used in this experiment were the same as those used in Experiment 1. Twenty slides were taken of each of the three real life events and 10 were selected to construct each story. In the new context condition, 4 slides were chosen for each story from the 10 slides not used in Experiment 1. These additional slides were termed belonging slides. Four slides out of each story were replaced by the four belonging slides in the test series for the new context condition. Each belonging slide fit in between two temporally contiguous slides in the original stories. It was randomly decided which of these two slides was replaced by the belonging slide.

Design and procedure. Experiment 3 employed a two-groups design. The correct context condition was replicated and was termed the second correct context group. In addition, a new context condition was created in which four slides out of each story were replaced by four belonging slides in the test sequence. This set of slides was arranged in the same random order and orientation as the test series for the second correct context condition. The independent variable was whether or not the subjects received belonging slides in the test series. The procedure was identical to the procedure in the first experiment. The subjects in the new context condition were not informed about the belonging slides and thus were forced to respond to them as if they had seen them before. Correct responses were scored with the belonging slides when subjects made orientation judgments that were appropriate for the flow of motion in the original stories.

\section{Results and Discussion}

The mean percent correct for the replicated correct context condition was $96.43 \%$; and the mean percent correct for the new context condition was $94.19 \%$. These may be compared with the original correct context condition in Experiment 1, where the percent correct was $95.33 \%$. There was no significant difference between the groups $[F(2,42)=.70, p>.50$, $\mathrm{MSe}=25.80 \mathrm{]}$. In addition, the new context group's performance with the belonging slides was compared to the second correct context group's performance with the replaced slides. There was no significant difference between the subjects' performance on these two sets of slides $[t(28)=.64, p>.50]$. The subjects in the new context condition reported the lateral orientation of the belonging slides so that they fit the context of the story, even though they had not seen them before. It appears that the subjects in these conditions apprehended the underlying events in such detail that knowledge of the underlying coherent events permitted them to specify the orientation of particular stimuli, even those previously unknown to them.

\section{GENERAL DISCUSSION}

This series of experiments provides further evidence against the notion that picture memory is in the form of sensory images. All the subjects in Experiment 1 viewed the same set of slides, yet their performance on the orientation recognition task was greatly influenced by whether they could extract coherent stories from the separate slides. If picture memory is in the form of exact images, the order and specific orientation of the pictures should not affect performance on the recognition task. It seems more likely that picture memory involves mental representations of the pictorial event which are neither verbal nor pictorial, but rather abstract and propositional (Pylyshyn, 1973). As evidenced by the subjects' lists of strategies for determining orientation in Experiment 1, the mental representations contained information about large salient objects, the relation of the figure to the background, and the activities of the people. In the conditions where the subjects could form three coherent stories with a consistent flow of movement, the mental representations were predominantly characterized by knowledge of the underlying stories and not the particular stimuli. In these conditions, the laterally reversed slides in the test series clashed with the meaningful pattern of the original events and the subjects could easily remember original orientation. Those subjects that could not form three coherent stories constructed mental representations that primarily contained specific features of the particular stimuli. The laterally reversed slides in the test series did not clash with their knowledge of the specific features and the reversed slide in the test series could not signal a meaningful alteration. In general, these experiments indicate that when pictorial material consists of stimuli whose orientation varies and when these variations differentially affect the events constructed by an observer, the orientation of this material will tend to be remembered. On the other hand, when pictorial material consists of stimuli whose orientation varies and when these variations have negligible effects on the events constructed by an observer, then the orientation of this material will tend not to be remembered.

\section{REFERENCE NOTES}

1. McCabe, L., \& Jenkins, J. J. Memory for pictures and words: Processing depends on interaction between tasks, materials, and criteria. Unpublished manuscript, 1977.

2. Kraft, R. N. The effect of imposed asymmetry on the discrimination of bilaterally symmetrical stimuli. Unpublished manuscript, 1975.

\section{REFERENCES}

Baggett, P. Memory for explicit and implicit information in picture stories. Journal of Verbal Learning and Verbal Behavior, 1975, 14, 538-548.

Bartlett, F. C. Remembering: $A$ study in experimental and social psychology. Cambridge: Cambridge University Press, 1932. P. 51.

Bower, G. H., \& KaRLIN, M. B. Depth of processing pictures of faces and recognition memory. Journal of Experimental Psychology, 1974, 4, 751-757.

Bransford, J. D., \& Franks, J. J. The abstraction of linguistic ideas. Cognitive Psychology, 1971, 2, 331-350.

Goldstein, A. G., \& Chance, J. E. Visual recognition memory for complex configurations. Perception \& Psychophysics, 1971, 9, 237-240.

Jenkins, J. J., Wald, J., \& Pittenger, J. B. Apprehending pictorial events: An instance of psychological cohesion. Minnesota Studies in the Philosophy of Science, in press. 
Klatzky, R. L., \& Stoy, A. M. Using visual codes for comparisons of pictures. Memory \& Cognition, 1974, 2, $727-736$.

Madigan, S., \& Rouse, M. Picture memory and visualgeneration processes. American Journal of Psychology, $1974,87,151-158$.

Nickerson, R. S. A note on long-term recognition memory for pictorial material. Psychonomic Science, 1968, 11, 58.

Pylyshyn, Z. W. What the mind's eye tells the mind's brain: A critique of mental imagery. Psychological Bulletin, 1973, 80, 1-24.
SHEPARD, R. N. Recognition memory for words, sentences, and pictures. Journal of Verbal Learning and Verbal Behavior, 1967, 6, 156-163.

Standing, L., Conezio, J., \& Haber, R. N. Perception and memory for pictures: Single-trial learning of 2,500 visual stimuli. Psychonomic Science, 1970, 19, 73-74.

(Received for publication February 10, 1977; revision accepted April 29, 1977.) 